OPEN ACCESS

Edited by:

Charles Christoph Roehr, University of Oxford, United Kingdom

Reviewed by: Marc Robin Mendler,

Ulm University Medical

Center, Germany

C. Omar Farouk Kamlin,

Royal Women's Hospital, Australia

*Correspondence:

Georg M. Schmölzer

georg.schmoelzer@me.com

Specialty section:

This article was submitted to

Neonatology,

a section of the journal

Frontiers in Pediatrics

Received: 22 August 2019 Accepted: 18 October 2019 Published: 05 November 2019

Citation:

Johnson PA, Morina N, O'Reilly M, Lee T-F, Cheung P-Y and Schmölzer GM (2019) Evaluation of a Tap-Based Smartphone App for Heart Rate Assessment During Asphyxia in a Porcine Model of Neonatal Resuscitation. Front. Pediatr. 7:453 doi: 10.3389/fped.2019.00453

\section{Evaluation of a Tap-Based Smartphone App for Heart Rate Assessment During Asphyxia in a Porcine Model of Neonatal Resuscitation}

\author{
Peter A. Johnson ${ }^{1,2}$, Nicolò Morina ${ }^{1,2}$, Megan O'Reilly ${ }^{1,2}$, Tze-Fun Lee ${ }^{1,2}$, Po-Yin Cheung ${ }^{1,2}$ \\ and Georg M. Schmölzer ${ }^{1,2 *}$ \\ ${ }^{1}$ Centre for the Studies of Asphyxia and Resuscitation, Neonatal Research Unit, Royal Alexandra Hospital, Edmonton, AB, \\ Canada, ${ }^{2}$ Department of Pediatrics, Faculty of Medicine and Dentistry, University of Alberta, Edmonton, AB, Canada
}

Objectives: Heart rate (HR) is the most significant parameter to assess a newborn's clinical status at birth. Recently, novel technologies including smartphone applications have been suggested for HR assessment during neonatal resuscitation. The aim of this study was to evaluate the accuracy, speed, and precision of the NeoTapLifeSupport (NeoTapLS) smartphone application using a digital stethoscope (DS) for HR assessment during neonatal resuscitation.

Design: Newborn piglets ( $n=20,1-3$ days, $1.7-2.4 \mathrm{~kg}$ ) were anesthetized, intubated, mechanically ventilated, and subjected to $30 \mathrm{~min}$ of hypoxia, followed by asphyxia. Asphyxia was induced by clamping the endotracheal tube and disconnecting the ventilator, until asystole was confirmed by zero carotid blood flow (CBF).

Setting: Experimental setting.

Subjects: Asphyxia-induced newborn piglets.

Interventions: During asphyxia, HR assessments were performed with a DS using the NeoTapLS smartphone application, and compared to 6-s method (6s), and 10-s method (10 s).

Measurements and Main Results: Accuracy of obtained HRs was compared to CBF and electrocardiogram and assessment time using NeoTapLS, $6 \mathrm{~s}$, and $10 \mathrm{~s}$ were also measured. The mean(SD) HR with the NeoTapLS was 68(26), compared to CBF with 68(27) bpm, $6 \mathrm{~s}$ with 68(27), and $10 \mathrm{~s}$ with 66(26) bpm during asphyxia. Bland-Altman analysis revealed no difference between HR using the NeoTapLS, $6 \mathrm{~s}, 10 \mathrm{~s}$, compared to CBF HR, with NeoTapLS showing the smallest difference between 95\% limits of agreement. The median (IQR) time required to obtain a HR using the NeoTapLS was $3(2-4)$ s, compared to $6(6-7)$, and $10(10-11)$ s for 6 and 10 s, respectively.

Conclusions: Our data suggests that the NeoTapLS is accurate, fast, and precise during neonatal asphyxia to assess heart rate.

Keywords: infants, newborn, neonatal resuscitation, heart rate, smartphone App, auscultation 


\section{INTRODUCTION}

The fetal-to-neonatal transition is a significant challenge at birth, which depends on effective lung aeration and onset of breathing $(1,2)$. The transition is a sequence of physiological events where liquid must be cleared from the lung, hemodynamic changes such as increase in pulmonary blood flow and systemic vascular resistance, occlusion of fetal shunts, and increases in heart rate (HR) occur (3-5). Asphyxia at birth is the most common reason that newborn infants fail to make a successful transition, as it can depress myocardial function and act against this sequence inducing bradycardia, which leads to asystole (cardiac arrest) (6). HR is therefore the most important parameter to assess a newborn infant's clinical status at birth. Assessment of HR is used to determine the timing, type and efficacy of respiratory support interventions that are needed during neonatal resuscitation $(7,8)$. It is imperative that $\mathrm{HR}$ be continuously monitored in newborns at risk for asphyxia, both rapidly and accurately. If HR detection is slow or overestimated, an intervention might be delayed or prolonged; alternatively, if HR is underestimated, it might result in inappropriate interventions.

The Neonatal Resuscitation Program (NRP) and European Resuscitation Council (ERC) Guidelines for Resuscitation recommends the combined use of pulse oximetry and electrocardiogram (ECG) for HR assessments throughout resuscitation (7-11). Pulse oximetry and ECG are routinely used to continuously monitor HR during neonatal resuscitation. However, on average it takes $\sim 90-120 \mathrm{~s}$ and $30-100 \mathrm{~s}$ to setup, detect and acquire a reliable HR signal after delivery using pulse oximetry and ECG, respectively (12-17). This is concerning because the first minutes of life are most critical for a newborn requiring assistance and when HR must be assessed quickly to decide what interventions are needed. Additionally, ECG and pulse oximetry are costly and might be inaccessible in resource-limited regions of the world. The NRP additionally recommends combining palpation and auscultation for HR assessment with pulse oximetry and ECG, while the ERC only suggests the use of palpation for assessing signs of recovery during resuscitation (9-11). While palpation and auscultation using a stethoscope can assess HR faster compared to ECG and pulse oximetry, these techniques notably underestimate HR by 8 and $13 \%$, respectively, when compared to ECG (18).

More recently, cost-effective and universally accessible assistive technology (e.g., tap-based smartphone and mobile apps) represent another approach to assess HR in newborn infants (16). Simulation studies reported that it is feasible to assess HR using tap-based smartphone applications (19-21). However, this technology has yet to be tested in vivo or during asphyxia in a neonatal model. The aim of this study was to assess the accuracy, speed, and precision of NeoTapLifeSupport (NeoTapLS), a tap-based smartphone application for HR

\footnotetext{
Abbreviations: HR, Heart rate; bpm, Beats per minute; ECG, Electrocardiography; PO, Pulse oximetry; $\mathrm{SpO}_{2}$, Oxygen saturation; NRP, Neonatal Resuscitation Program; ERC, European Resuscitation Council; CBF, Carotid blood flow; NeoTapLS, NeoTapLifeSupport app; 6sec, 6-sec method; 10sec, 10-sec method; SD, Standard deviation; IQR, Interquartile range.
}

assessment during asphyxia in our porcine model of neonatal resuscitation for the first time. We hypothesized that NeoTapLS would have a similar accuracy compared to HR assessed by carotid blood flow (CBF).

\section{MATERIALS AND METHODS}

Twenty newborn mixed breed piglets (1-3 days of age, weighing $2.0 \pm 0.4 \mathrm{~kg}$ ) were obtained on the day of experimentation from the University Swine Research Technology Center. All experiments were conducted in accordance with the guidelines and approval of the Animal Care and Use Committee (Health Sciences), University of Alberta (AUP2151), presented according to the ARRIVE guidelines (22), and registered at preclincialtrials.eu (PCTE155).

\section{Animal Preparation}

Piglets were instrumented as previously described with modifications (23-26). Following the induction of anesthesia using isoflurane, piglets were intubated via a tracheostomy, and pressure-controlled ventilation (Acutronic Fabian HFO; Hirzel, Switzerland) was commenced at a respiratory rate of 16-20 breaths/min and pressure of $20 / 5 \mathrm{cmH}_{2} \mathrm{O}$. Oxygen saturation was kept within $90-100 \%$, glucose level and hydration was maintained with an intravenous infusion of 5\% dextrose at 10 $\mathrm{mL} / \mathrm{kg} / \mathrm{h}$. During the experiment anesthesia was maintained with intravenous propofol 5-10 mg/kg/h and morphine $0.1 \mathrm{mg} / \mathrm{kg} / \mathrm{h}$. Additional doses of propofol (1-2 mg/kg) and morphine (0.05$0.1 \mathrm{mg} / \mathrm{kg}$ ) were given as needed. The piglet's body temperature was maintained at $38.5-39.5^{\circ} \mathrm{C}$ using an overhead warmer and a heating pad.

\section{Haemodynamic Parameters}

A 5-French Argyle ${ }^{\circledR}$ (Klein-Baker Medical Inc. San Antonio, TX) double-lumen catheter was inserted via the right femoral vein for administration of fluids and medications. A 5-French Argyle ${ }^{\circledR}$ single-lumen catheter was inserted above the right renal artery via the femoral artery for continuous arterial blood pressure monitoring in addition to arterial blood gas measurements. The right common carotid artery was also exposed and encircled with a real-time ultrasonic flow probe $(2 \mathrm{~mm}$; Transonic Systems Inc., Ithica, NY) to measure CBF. Piglets were placed in supine position and allowed to recover from surgical instrumentation until baseline haemodynamic measures were stable (minimum of $1 \mathrm{~h}$ ). Ventilator rate was adjusted to keep the partial arterial $\mathrm{CO}_{2}$ between 35 and 45 torr as determined by periodic arterial blood gas analysis. Mean systemic arterial pressure, systemic systolic arterial pressure, HR, and percutaneous oxygen saturation were continuously measured and recorded throughout the experiment with a Hewlett Packard 78833B monitor (Hewlett Packard Co., Palo Alto, CA) and LabChart ${ }^{\circledR}$ programming software (ADInstruments, Houston, TX, United States).

\section{ECG and CBF}

A 3-lead ECG was placed on skin at the right fore limb, left fore limb and left hind limb. In addition to its use for HR monitoring, CBF can also be utilized to calculate HR. While ECG HR is 
commonly used as a clinical gold standard, CBF HR was selected as the experimental gold standard, which offers a comparatively better measure of HR in the setting of asphyxia and in the likely event of pulseless electrical activity (27). However, both ECG and $\mathrm{CBF}$ were used as standard for HR comparisons.

\section{Digital Stethoscope}

The digital stethoscope (DS) is a form of electronic stethoscope, which functions by converting the audial heartbeat signal into an electronic signal followed by amplification to provide clearer detection $(16,28)$. Several studies report the DS is not affected by the use of respiratory support and can be utilized for HR assessment in newborn infants with better accuracy, compared to a standard stethoscope $(16,29,30)$.

Auscultation was performed using a DS (Thinklabs One, Denver, CO). Assessments were performed using both the (i) 6 -s method $(6 \mathrm{~s})$ and ii) 10 -s method $(10 \mathrm{~s})$. The $6 \mathrm{~s}$ method is currently recommended by the NRP (11), whereby HR is calculated by multiplying the number of heartbeats heard in $6 \mathrm{~s}$ by 10 . The $10 \mathrm{~s}$ method has previously been recommended for initial HR assessment at birth $(31,32)$, and calculates HR by multiplying the number of heartbeats heard in $10 \mathrm{~s}$ by 6 . The frequency filter of the DS was set between 30 and $500 \mathrm{~Hz}$, which produces low frequency heart sounds and filters out lung sounds, and amplification was set to 6 on the $0-10$ Scale.

\section{Tap-Based Smartphone App}

The NeoTapLifeSupport (NeoTapLS; Tap4Life, Stockholm, Sweden) smartphone app was downloaded from App Store (Apple, Cupertino, CA) and paired with the digital stethoscope for HR assessments. NeoTapLS is a recent development for HR assessment, however has only been tested in high-fidelity resuscitation simulation scenarios (16, 19-21). NeoTapLS displays a HR generated by at least three taps on the smartphone screen, which coincides with what the healthcare provider auscultates. Based on this predefined calculation algorithm, when $\mathrm{HR}$ is $30 \mathrm{bpm}$, a minimum of $6 \mathrm{~s}$ is required to assess HR $\left[3^{*}(60 / 30)=6 \mathrm{~s}\right]$, and at $18 \mathrm{bpm}$, it will take a minimum of $10 \mathrm{~s}$ $\left[3^{*}(60 / 18)=10 \mathrm{~s}\right]$. Thus, it was expected to be faster than the 6 and $10 \mathrm{~s}$ method on average. The time needed to display HR from the first tap until the app assessed HR was recorded.

\section{Experimental Protocol}

Following at least $1 \mathrm{~h}$ of stabilization after the surgical protocol, piglets were subjected to $30 \mathrm{~min}$ of nitrogen-induced hypoxia $\left(\mathrm{FiO}_{2} 10-15 \%\right)$. Hypoxia was then followed by asphyxia until asystole, achieved by disconnecting the ventilator and clamping the endotracheal tube. Asystole was defined as no audible HR during auscultation for at least $10 \mathrm{~s}$ and zero CBF. All HR assessments were performed during the asphyxia time leading to asystole (i.e., between disconnecting the ventilator and clamping the endotracheal tube and confirmation of asystole) and were performed by a single investigator (GMS), who was blinded to HR displayed by ECG and CBF. HR assessments comprised of auscultation using the DS in three different methods: (i) NeoTapLS, (ii) $6 \mathrm{~s}$, and (iii) $10 \mathrm{~s}$. All NeoTapLS, 6 and $10 \mathrm{~s}$ HR assessments were assessed from the same starting time point.
For NeoTapLS, GMS simultaneously tapped the smartphone screen for each auscultated heartbeat, and the displayed HR was recorded by PAJ. For 6 and $10 \mathrm{~s}$, the number of heartbeats auscultated was verbalized by the assessor (GMS) at 6 and $10 \mathrm{~s}$ and recorded by PAJ. GMS was not required to perform arithmetic for determination of HR in bpm; this was determined independently during data analysis. These assessments were repeatedly performed every $30 \mathrm{~s}$ in all piglets during asphyxia until asystole. This enabled HR assessment to be performed at various levels of bradycardia, which are representative of different clinical situations (i.e., HR $>100$, between 60 and 100, or $<60 \mathrm{bpm}$ ) (11). Markers were placed within the LabChart program to indicate HR assessment times. Post-experiment, the marker was then compared to waveforms from the ECG and CBF to determine HR at the time of assessment using 6, $10 \mathrm{~s}$, and NeoTapLS. HR as determined by CBF was defined as the gold standard (33). Following confirmation of asystole, HR assessments were ceased and interventions were performed according to the study protocol (23).

\section{Statistical Analysis}

All statistical analyses were performed by a statistician who was blinded to the HR assessment approach. Using a single assessor (GMS) eliminated any user bias and error caused by variations between assessors while concurrently allowing for comparison of the same $\mathrm{HR}$ at a given point in time. Results from HR assessments are presented as mean (SD). The level of agreement between the measured HR for NeoTapLS, 6 and 10 s intervention groups and CBF HR were assessed using Bland-Altman plots (34). To determine differences at varying HRs during asphyxia, assessments were clustered into subgroups based on CBF HR. A priori subgroups were defined as per NRP HR cut-offs (11) HR $<60,60-100$, and $>100 \mathrm{bpm}$. Time to assess NeoTapLS, 6 and $10 \mathrm{~s}$ are presented as median (IQR). The data was tested for normality and compared using one-way ANOVA with Bonferoni post-test. $P$-values are two-sided and $p<0.05$ was considered statistically significant. Statistical analyses were performed with Stata (StataCorp, College Station, TX).

\section{RESULTS}

Twenty newborn mixed breed piglets were obtained on the day of the experiment; baseline data and pre-asphyxia parameters are presented in Table 1. The median (IQR) number of assessments per animal was $11(2-20)$ observations. A total of $138 \mathrm{HR}$ assessments were performed during asphyxia with 16 observations $>100$ bpm, 68 observations between 60 and 100 bpm, and 54 observations $<60 \mathrm{bpm}$.

The mean(range) time for asphyxia was 404(72-600) s During asphyxia, the mean (SD) CBF and ECG HR were 68 (27) and 68 (27) bpm, respectively. The HR using NeoTapLS, 6 and $10 \mathrm{~s}$ methods were 68 (27), 66 (26), and 68 (26) bpm, respectively (Figure 1). There were no significant differences in the mean(SD) HR measured using CBF, ECG, NeoTapLS, 6 and $10 \mathrm{~s}$ methods. However, 95\% upper and lower limits of agreement varied for each technique. The Bland-Altman comparisons for $\mathrm{CBF}$ HR vs. 6 or $10 \mathrm{~s}$ or NeoTapLS are displayed in Figures 2A-C, 
TABLE 1 | Baseline and pre-asphyxia parameters.

\begin{tabular}{|c|c|}
\hline$n$ & 20 \\
\hline \multicolumn{2}{|l|}{ SEX } \\
\hline Female & 7 \\
\hline Male & 13 \\
\hline Weight $(\mathrm{kg})^{\dagger}$ & $2.08(1.8-2.2)$ \\
\hline Age (days) $^{\dagger \dagger}$ & $1.85(1-3)$ \\
\hline \multicolumn{2}{|l|}{ BASELINE } \\
\hline $\mathrm{SpO}_{2}(\%)$ & $98.8(97-99)$ \\
\hline Heart rate (bpm) & 175 (160-204) \\
\hline MAP (mm Hg) & $59.2(55-71)$ \\
\hline CVP (mm Hg) & $3.9(2-5)$ \\
\hline $\mathrm{pH}$ & $7.52(7.4-7.6)$ \\
\hline $\mathrm{PaCO}_{2}$ (torr) & $36.1(32.8-40.6)$ \\
\hline $\mathrm{PaO}_{2}$ (torr) & $102.5(81-130)$ \\
\hline BEcf (mmol/L) & $4.6(0-6)$ \\
\hline $\mathrm{HCO}_{3}(\mathrm{mmol} / \mathrm{L})$ & $30.09(24.2-32.81)$ \\
\hline \multicolumn{2}{|l|}{ PRE-ASPHYXIA } \\
\hline $\mathrm{SpO}_{2}(\%)$ & $30.4(15-48)$ \\
\hline Heart rate (bpm) & 256 (187-277) \\
\hline MAP $(m m ~ H g)$ & $50.8(36-64)$ \\
\hline CVP (mm Hg) & $4.6(2-5)$ \\
\hline $\mathrm{pH}$ & $6.57(6.5-6.7)$ \\
\hline $\mathrm{PaCO}_{2}$ (torr) & $100.5(88-113)$ \\
\hline $\mathrm{PaO}_{2}$ (torr) & $14.1(11-19)$ \\
\hline BEcf (mmol/L) & $-28.8(-30$ to -26$)$ \\
\hline $\mathrm{HCO}_{3}(\mathrm{mmol} / \mathrm{L})$ & $9.16(6.9-11.4)$ \\
\hline
\end{tabular}

Data are presented as median (IQR) unless indicated ${ }^{\dagger}$ mean (SD) or ${ }^{\dagger+}$ mean (range).

respectively. Analyses by HR cutoff ranges: $<60,60-100$, and $>100 \mathrm{bpm}$ are presented in Figure 3.

The median(IQR) time needed to assess HR during asphyxia using the NeoTapLS, 6 and $10 \mathrm{~s}$ was 3(2-4), 6(6-7), and $10(10-11) \mathrm{s}$.

\section{DISCUSSION}

To our knowledge, this is the first study to compare different methods for HR assessment during neonatal asphyxia. Overall, the NeoTapLS had similar accuracy of HR assessment compared to the $6 \mathrm{~s}$ method, $10 \mathrm{~s}$ methods, ECG, and CBF. Furthermore, subgroup analysis by NRP cut-off values showed similar accuracy of HR assessment. Our study suggests that all evaluated methods have similar accuracy for HR assessments in our asphyxia model. Furthermore, the NeoTapLS method was the fastest technique to assess HR. Our results suggest that the NeoTapLS application might be a useful tool in combination with a stethoscope to assess HR at birth.

In the current study, we observed similar accuracy in HR assessment between the DS, ECG, and CBF. However, there are conflicting results about the accuracy of using the DS to assess a newborn's HR. In the Neonatal Intensive Care Unit and the delivery room, HR assessment using ECG or DS have previously demonstrated low and high accuracy for DS with

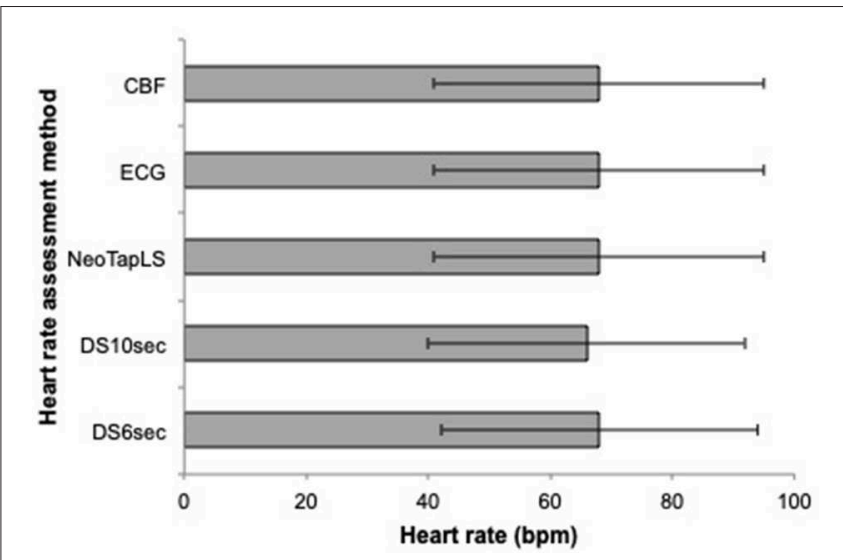

FIGURE 1 | Mean (SD) heart rate during asphyxia assessed using the 6, $10 \mathrm{~s}$, NeoTapLS interventions and standards (ECG and CBF). NeoTapLS,

Assessment intervention group using the NeoTapLS smartphone app paired with the digital stethoscope; DS6s, Assessment intervention group with the 6-s method using a digital stethoscope; DS10 s, Assessment intervention group with the 10-s method using a digital stethoscope; ECG,

electrocardiogram; CBF, carotid blood flow.

mean differences of 7.4 and $0.2 \mathrm{bpm}$, respectively $(12,29)$. Furthermore, Gaertner et al. reported that the DS only detected HR in 23/37 newborn infants within $30 \mathrm{~s}$ (30). In the remaining 14 infants HR could not be assessed due to crying (30). In the current study, all piglets were intubated and sedated/anesthetized and therefore there was no crying/vocalization, which might result in a clearer HR assessment. Moreover, in a real-life resuscitation scenario, crying/vocalization would be unlikely under asphyxia and instead a sign of improved status, where HR assessment and resuscitative interventions might no longer be necessary.

Overall, HR assessment using auscultation has been reported to be inaccurate in $33-75 \%$ of cases $(20,35,36)$. This could be due to the mental computation required to convert heartbeat counts to HR; however, tap-based applications might reduce these inaccuracies. Furthermore, we speculate time needed to assess HR will take longer in a clinical resuscitation scenario with $10 \mathrm{~s}$ as a result of greater cognitive load required for multiplying numbers by 6 , in contrast to the $6 \mathrm{~s}$ where numbers can easily be multiplied by 10 .

Two studies reported that the NeoTapLS application has good accuracy and can be used to quickly assess HR in combination with auscultation during simulated neonatal resuscitation (19, 20). These studies suggest that tap-based mobile applications might have the potential to improve HR assessment in the delivery room. However, studies in the delivery room are lacking. The current study was the first to assess HR during neonatal asphyxia. We observed that the NeoTapLS application paired with the DS had similar accuracy in assessing HR compared to the DS (6- and 10-s rule), ECG, or CBF. Additionally, the NeoTapLS had a shorter median assessment time compared to the 6 and 10$s$ rule. Since we did not include the time needed for performing mental arithmetic for the 6 and 10-s rule, we speculate the net time for $\mathrm{HR}$ assessment will be much longer. Our results suggest 

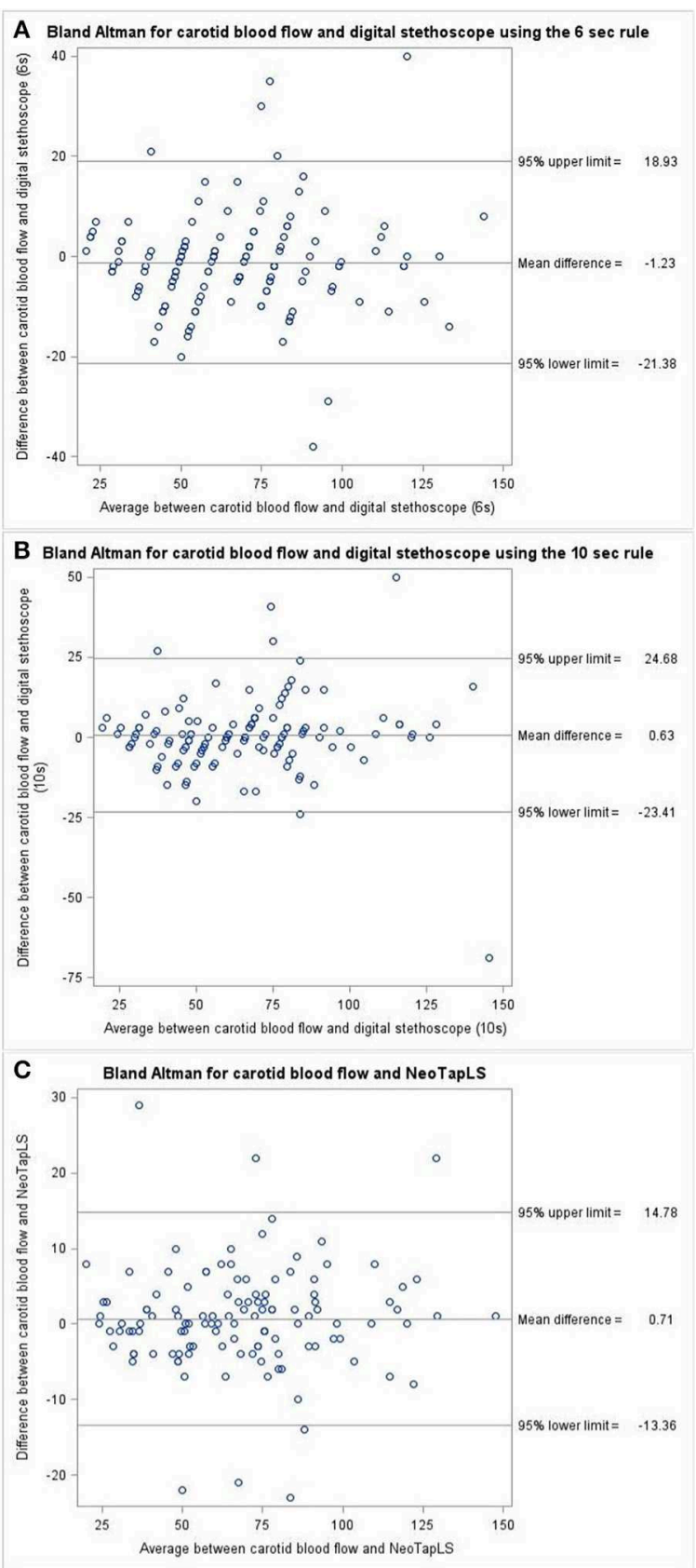

FIGURE 2 | Bland-Altman plot for (A) DS6 s vs. CBF, (B) DS10 s vs. CBF, and (C) NeoTapLS vs. CBF heart rate assessments during asphyxia. CBF, carotid blood flow; DS6 s, digital stethoscope using 6 s method; DS10 s, digital stethoscope using 10 s method; Digital stethoscope paired with NeoTapLS app.

that the NeoTapLS application might be useful during neonatal asphyxia to assess HR.

Our Bland-Altman comparisons identified varying upper and low limits of $\mathrm{HR}$ assessment using each technique, when compared to the CBF HR. Although all techniques were comparable to the gold standard (CBF HR), NeoTapLS demonstrated the greatest precision for HR assessment as shown

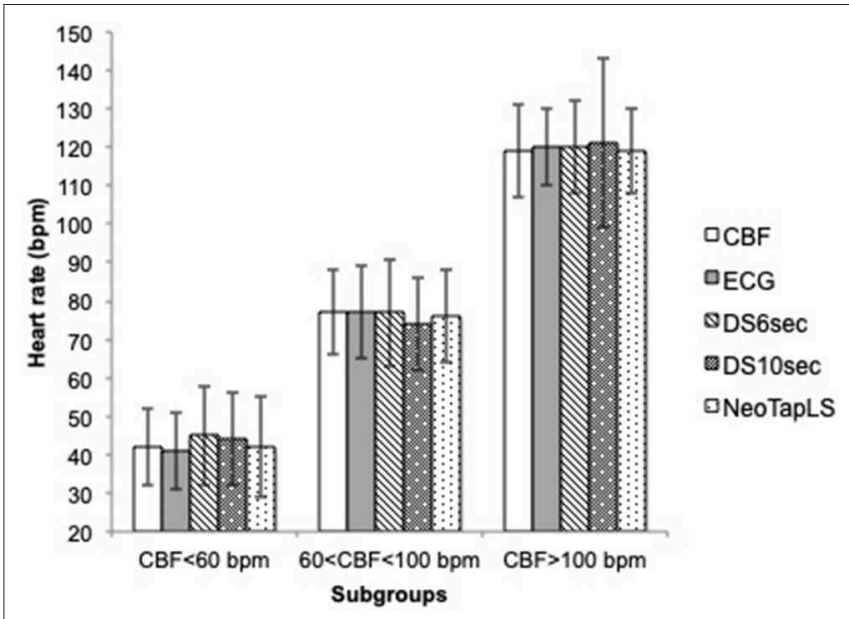

FIGURE 3 | Mean (SD) heart rate during asphyxia assessed by CBF, ECG, DS6 s, DS10 s, and NeoTapLS, according to subgroup 1 (CBF<60 bpm), 2 (60<CBF<100 bpm), and $3(\mathrm{CBF}>100 \mathrm{bpm})$. DS6 s, DS10 s, NeoTapLS interventions and standards (ECG and CBF). DS6 s, Assessment intervention group with the 6-s method using a digital stethoscope; DS10 s, Assessment intervention group with the 10-s method using a digital stethoscope;

NeoTapLS, Assessment intervention group using the NeoTapLS smartphone app paired with the digital stethoscope; ECG, electrocardiogram; CBF, carotid blood flow.

by the least difference between 95\% upper and lower limits of agreement. This suggests that NeoTapLS offers the higher precision for HR assessment, compared to 6 and $10 \mathrm{~s}$. Despite this, there is persisting variability with NeoTapLS compared to CBF with a wide difference in 95\% upper and lower limits of agreement.

We have also identified several limitations for this technology. First, it is only possible to make assessments using both hands, one hand to hold the stethoscope and the other to use NeoTapLS, which means one member of the clinical team must be dedicated for each time HR assessments are performed. Additionally, when using a mobile phone in the delivery room, HR assessments should be the sole purpose and it should be ensured it is thoroughly disinfected before and after use.

\section{LIMITATIONS}

Our newborn piglet model is a great strength of this translational study, as this model closely simulates delivery room events, with the gradual onset of severe asphyxia leading to bradycardia and cardiac arrest. However, a few limitations should be considered before implementing these methods in the delivery room. Our asphyxia model uses piglets that were sedated/anesthetized, which is not the case in clinical settings. Our study did not require the assessor to perform mental arithmetic to calculate the HR based off of heartbeat counts when using the 6- and 10 -s method; instead the assessor verbally reported the heartbeat count value at 6- and 10-s As the aim for the current study was to compare each HR assessments technique independently, performing the assessment by single assessor (GMS) eliminated user bias and error caused by variations between assessors, 
while allowing for comparison of the same HR. However, this limits the generalizability of this study to assessors at different skill levels and does not account for time needed to calculate HR following auscultation. Additionally, the current study utilizes the DS together with all methods, which may differ in accuracy compared to these techniques combined with a standard stethoscope. While the DS was used as a standardized tool in this study, it may not readily be available for use in resource-limited regions, as well. Another limitation of our study is that $\mathrm{HR}<30 \mathrm{bpm}$ was infrequent and thus, difficult to assess accuracy and speed of assessments at extremely low HRs.

\section{CONCLUSION}

Heart rate assessment with the NeoTapLS application had similar accuracy compared to auscultation with a digital stethoscope with the 6-s method, 10-s method, the electrocardiogram or the carotid blood flow during neonatal asphyxia. The NeoTapLS application had higher precision and a faster time to assess heart rate compared to the 6- or 10-s method with a digital stethoscope. However, clinical trials to evaluate the utility of tap-based applications during neonatal resuscitation are warranted.

\section{DATA AVAILABILITY STATEMENT}

The datasets generated for this study are available on request to the corresponding author.

\section{ETHICS STATEMENT}

The animal study was reviewed and approved by Approval of the Animal Care and Use Committee (Health Sciences), University

\section{REFERENCES}

1. Hooper SB, Polglase GR, Roehr CC. Cardiopulmonary changes with aeration of the newborn lung. Paediatr Respir Rev. (2015) 16:147-50. doi: 10.1016/j.prrv.2015.03.003

2. Hooper SB, Kitchen MJ, Polglase GR, Roehr CC, Te Pas AB. The physiology of neonatal resuscitation. Curr Opin Pediatr. (2018) 30:187-91. doi: 10.1097/MOP.0000000000000590

3. Hooper SB, Te Pas AB, Lang J, van Vonderen JJ, Roehr CC, Kluckow M, et al. Cardiovascular transition at birth: a physiological sequence. Pediatr Res. (2015) 77:608-14. doi: 10.1038/pr.2015.21

4. van Vonderen JJ, Roest AA, Siew ML, Blom NA, van Lith JM, Walther FJ, et al. Noninvasive measurements of hemodynamic transition directly after birth. Pediatr Res. (2014) 75:448-52. doi: 10.1038/pr.2013.241

5. van Vonderen JJ, Roest AA, Siew ML, Walther FJ, Hooper SB, te Pas AB. Measuring physiological changes during the transition to life after birth. Neonatology. (2014) 105:230-42. doi: 10.1159/000356704

6. Kapadia V, Wyckoff MH. Chest compressions for bradycardia or asystole in neonates. Clin Perinatol. (2012) 39:833-42. doi: 10.1016/j.clp.2012.09.011

7. Wyckoff M, Aziz K, Escobedo M, et al. Part 13: Neonatal Resuscitation 2015 American Heart Association Guidelines Update for Cardiopulmonary Resuscitation and Emergency Cardiovascular Care. (2015). Available online at: www.pediatrics.org/cgi/doi/10.1542/peds.2015--3373G doi: 10.1542/peds.2015-3373G of Alberta (AUP00002151), presented according to the ARRIVE guidelines, and registered at preclincialtrials.eu (PCTE0000155).

\section{AUTHOR CONTRIBUTIONS}

GS, PY-C, and MO'R: conception. GS, NM, T-FL, MO'R, P-YC, and PJ: data acquisition, data analysis, interpreting of results, drafting of the manuscript, critical revision of the manuscript, and final approval of the manuscript.

\section{FUNDING}

The study was supported by a Grant from the SickKids Foundation in partnership with the Canadian Institutes of Health Research [CIHR - Institute of Human Development, Child and Youth Health (IHDCYH)], New Investigator Research Grant Program (Grant No. NI17-033). This research has been facilitated by the Women and Children's Health Research Institute through the generous support of the Stollery Children's Hospital Foundation.

\section{ACKNOWLEDGMENTS}

We would like to thank the public for donating money to our funding agencies: PJ was a recipient of the University of Alberta Faculty of Medicine \& Dentistry Medical Sciences Graduate Program Scholarship and the Women \& Children's Health Research Institute Graduate Studentship Award. GS was a recipient of the Heart and Stroke Foundation/University of Alberta Professorship of Neonatal Resuscitation, a National New Investigator of the Heart and Stroke Foundation Canada and an Alberta New Investigator of the Heart and Stroke Foundation Alberta.

8. Wyllie J, Bruinenberg J, Roehr CC, Rüdiger M, Trevisanuto D, Urlesberger B. European Resuscitation Council Guidelines for Resuscitation 2015. Section 7. Resuscitation and support of transition of babies at birth. Resuscitation. (2015) 95:249-63. doi: 10.1016/j.resuscitation.2015.07.029

9. Perlman J, Wyllie J, Kattwinkel J, Wyckoff MH, Aziz K, Guinsburg R, et al. Part 7: neonatal resuscitation 2015 international consensus on cardiopulmonary resuscitation and emergency cardiovascular care science with treatment recommendations. Circulation. (2015) 132 (Suppl:S204-S241). doi: 10.1161/CIR.0000000000000276

10. Monsieurs KRG, Nolan JP, Bossaert LL, Greif R, Maconochie IK, Nikolaou NI, et al. European resuscitation council guidelines for resuscitation 2015. Section 1. Executive summary. Resuscitation. (2015) 95:1-80. doi: 10.1016/j.resuscitation.2015.07.015

11. Weiner GM, Zaichkin J. Textbook of Neonatal Resuscitation. American Academy of Pediatrics (2016). Available online at: http://ebooks. aappublications.org/content/textbook-of-neonatal-resuscitation-nrp7th-ed (accessed January 8, 2019).

12. Treston BP, Semberova J, Kernan R, Crothers E, Branagan A, O'Cathain $\mathrm{N}$, et al. Assessment of neonatal heart rate immediately after birth using digital stethoscope, handheld ultrasound and electrocardiography: an observational cohort study. Arch Dis Child Fetal Neonatal. (2018) 104:F227 doi: 10.1136/archdischild-2018-315619

13. Mizumoto H, Tomotaki S, Shibata H, Ueda K, Akashi R, Uchio H, et al. Electrocardiogram shows reliable heart rates much earlier than pulse 
oximetry during neonatal resuscitation. Pediatr Int. (2012) 54:205-207. doi: 10.1111/j.1442-200X.2011.03506.x

14. van Vonderen JJ, Hooper SB, Kroese JK, Roest AA, Narayen IC, van Zwet EW, et al. Pulse oximetry measures a lower heart rate at birth compared with electrocardiography. J Pediatr. (2015) 166:49-53. doi: 10.1016/j.jpeds.2014.09.015

15. Katheria A, Arnell K, Brown M, Hassen K, Maldonado M, Rich W, et al. A pilot randomized controlled trial of EKG for neonatal resuscitation. PLoS ONE. (2017) 12:e0187730. doi: 10.1371/journal.pone.0187730

16. Johnson PA, Cheung PY, Lee TF, O'Reilly M, Schmölzer GM. Novel technologies for heart rate assessment during neonatal resuscitation at birth - a systematic review. Resuscitation. (2019). doi: 10.1016/j.resuscitation.2019.07.018

17. Roehr CC, O'Shea JE, Dawson JA, Wyllie JP. Devices used for stabilisation of newborn infants at birth. Arch Dis Child Fetal Neonatal Ed. (2018) 103:F66-71. doi: 10.1136/archdischild-2016-310797

18. Kamlin CO, O’Donnell CP, Everest NJ, Davis PG, Morley CJ. Accuracy of clinical assessment of infant heart rate in the delivery room. Resuscitation. (2006) 71:319-21.doi: 10.1016/j.resuscitation.2006.04.015

19. Myrnerts Höök S, Pejovic NJ, Marrone G, Tylleskär T, Alfvén T. Accurate and fast neonatal heart rate assessment with a smartphone-based application-a manikin study. Acta Paediatr. (2018). doi: 10.1111/apa.14350

20. Binotti M, Cavallin F, Ingrassia PL, Pejovic NJ, Monzani A, Genoni $\mathrm{G}$, et al. Heart rate assessment using NeoTapAdvancedSupport: a simulation study. Arch Dis Child Fetal Neonatal Ed. (2018) 104:1-3. doi: 10.1136/archdischild-2018-315408

21. Cavallin F, Binotti M, Ingrassia PL, Genoni G, Rizzollo S, Monzani A, et al. Impact of a mobile application for heart rate assessment in simulated neonatal resuscitation: a randomised controlled cross-over study. Arch Dis Child - Fetal Neonatal Ed. (2019). doi: 10.1136/archdischild-2018-316757

22. Kilkenny C, Browne WJ, Cuthill IC, Emerson M, Altman DG. Improving bioscience research reporting: the ARRIVE guidelines for reporting animal research. PLoS Biol. (2010) 8:e1000412. doi: 10.1371/journal.pbio.1000412

23. Mustofa J, Cheung PY, Patel S, Lee TF, Lu M, Pasquin MP, et al. Effects of different durations of sustained inflation during cardiopulmonary resuscitation on return of spontaneous circulation and hemodynamic recovery in severely asphyxiated piglets. Resuscitation. (2018) 129:82-9. doi: 10.1016/j.resuscitation.2018.06.013

24. Schmölzer GM, O’Reilly M, Labossiere J, Lee TF, Cowan S, Nicoll J, et al. 3:1 Compression to ventilation ratio versus continuous chest compression with asynchronous ventilation in a porcine model of neonatal resuscitation. Resuscitation. (2014) 85:270-5. doi: 10.1016/j.resuscitation.2013. 10.011

25. Schmölzer GM, O’Reilly M, Labossiere J, Lee TF, Cowan S, Qin $\mathrm{S}$, et al. Cardiopulmonary resuscitation with chest compressions during sustained inflations. Circulation. (2013) 128:2495-503. doi: 10.1161/CIRCULATIONAHA.113.002289

26. Cheung P-Y, Gill RS, Bigam DL. A swine model of neonatal asphyxia. J Vis Exp. (2011) 2011:3166. doi: 10.3791/3166

27. Solevåg AL, Luong D, Lee TF, O’Reilly M, Cheung PY, Schmölzer GM. Nonperfusing cardiac rhythms in asphyxiated newborn piglets [Internet]. PLoS ONE. (2019) 14:e0214506. doi: 10.1371/journal.pone.0214506

28. Swarup S, Makaryus AN. Digital stethoscope: technology update. Med Dev. (2018) 11:29-36. doi: 10.2147/MDER.S135882

29. Kevat AC, Dawson J, Davis PG, Kamlin CO. Evaluation of a digital stethoscope and smart device technology for assessment of heart rate in the newborn infant [Internet]. Arch Dis Child Fetal Neonatal Edt. (2015) 100:F562-3. doi: 10.1136/archdischild-2015-308639

30. Gaertner VD, Kevat AC, Davis PG, Kamlin COF. Evaluation of a digital stethoscope in transitioning term infants after birth. [Internet]. Arch Dis Child Fetal Neonatal Ed. (2017) 102:F370-1. doi: 10.1136/archdischild-2016-312316

31. Wilson F, Park W. Basic Resuscitation and Primary Care. Lancaster: Springer Science \& Business Media (2012).

32. Kobayashi H. Effect of measurement duration on accuracy of pulse-counting. Ergonomics. (2013) 56:1940-4. doi: 10.1080/00140139.2013.840743

33. Luong DH, Cheung P-Y, O’Reilly M, Lee TF, Schmolzer GM. Electrocardiography vs. auscultation to assess heart rate during cardiac arrest with pulseless electrical activity in newborn infants. Front Pediatr. (2018) 6:1-4. doi: 10.3389/fped.2018.00366

34. Bland JM, Altman DG. Statistical methods for assessing agreement between two methods of clinical measurement. Lancet. (1986) 1:307-10. doi: 10.1016/S0140-6736(86)90837-8

35. Wyckoff MH, Aziz K, Escobedo MB, Kapadia VS, Kattwinkel J, Perlman JM, et al. Part 13: neonatal resuscitation. Circulation. (2015) 132:S543-60. doi: 10.1161/CIR.0000000000000267

36. Voogdt KG, Morrison AC, Wood FE, van Elburg RM, Wyllie JP. A randomised, simulated study assessing auscultation of heart rate at birth. Resuscitation. (2010) 81:1000-3. doi: 10.1016/j.resuscitation.2010.03.021

Conflict of Interest: The authors declare that the research was conducted in the absence of any commercial or financial relationships that could be construed as a potential conflict of interest.

Copyright (C) 2019 Johnson, Morina, O'Reilly, Lee, Cheung and Schmölzer. This is an open-access article distributed under the terms of the Creative Commons Attribution License (CC BY). The use, distribution or reproduction in other forums is permitted, provided the original author(s) and the copyright owner(s) are credited and that the original publication in this journal is cited, in accordance with accepted academic practice. No use, distribution or reproduction is permitted which does not comply with these terms. 